\title{
Contents
}

\section{Part I Preamble}

Republication of: Radial Movement in Sunspots ....................... 2

S.S. Hasan

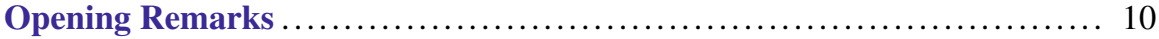

E. Priest

Solar Physics at the Kodaikanal Observatory:

A Historical Perspective

S.S. Hasan, D.C.V. Mallik, S.P. Bagare, and S.P. Rajaguru

Vainu Bappu Memorial Lecture: What is a Sunspot?

D.O. Gough

\section{Part II Oral Presentations}

Angular-Momentum Coupling Through the Tachocline.

68

D.O. Gough

Outstanding Issues in Solar Dynamo Theory ....................... 86

D. Nandy

Status of 3D MHD Models of Solar Global Internal Dynamics ........... 96

A.S. Brun

Measuring the Hidden Aspects of Solar Magnetism

J.O. Stenflo

Recent Advances in Chromospheric and Coronal Polarization

Diagnostics

J. Trujillo Bueno 
Probability Density Functions to Represent Magnetic Fields at the Solar Surface

M. Sampoorna

Spectropolarimetry with CRISP at the Swedish 1-m Solar

Telescope

A. Ortiz and L.H.M. Rouppe van der Voort

Spectropolarimetry with the NLST

K. Sankarasubramanian, S.S. Hasan, and K.E. Rangarajan

Magnetic Coupling in the Quiet Solar Atmosphere 166

O. Steiner

The Evershed Effect with SOT/Hinode 186

$\mathrm{K}$. Ichimoto and the SOT/Hinode Team

The Evershed Flow and the Brightness of the Penumbra 193

L.R. Bellot Rubio

A Topology for the Penumbral Magnetic Fields

J. Sánchez Almeida

Theoretical Models of Sunspot Structure and Dynamics

J.H. Thomas

Convection and the Origin of Evershed Flows

Å. Nordlund and G.B. Scharmer

The Magnetic Field of Solar Spicules

R. Centeno, J. Trujillo Bueno, and A. Asensio Ramos

Three-Dimensional Magnetic Reconnection

C.E. Parnell and A.L. Haynes

Signatures of Coronal Heating Mechanisms

P. Antolin, K. Shibata, T. Kudoh, D. Shiota, and D. Brooks

Waves in Polar Coronal Holes

D. Banerjee

MHD Wave Heating Diagnostics

Y. Taroyan and R. Erdélyi 
Coronal Mass Ejections from Sunspot and Non-Sunspot

Regions

N. Gopalswamy, S. Akiyama, S. Yashiro, and P. Mäkelä

CME Observations from STEREO 308

N. Srivastava

Low-Frequency Radio Observations of Coronal Magnetic

Fields

R. Ramesh, S.M. Sonnett, and C. Kathiravan

Evolution of Near-Sun Solar Wind Turbulence.

P.K. Manoharan

The Solar-Stellar Connection

J.H.M.M. Schmitt

Summary and Perspective.

N.O. Weiss

\section{Part III Poster Presentations}

Evidence for Return Meridional Flows in the Convection Zone

K.R. Sivaraman, H. Sivaraman, S.S. Gupta, and R.F. Howard

Long-Term Variations in Meridional Flows

J. Javaraiah

Low-Degree High-Frequency $p$ and $g$ Modes in the Solar Core

S. Mathur, R.A. García, and A. Eff-Darwich

Interior and Exterior Clues of Solar Activity

S. Turck-Chièze, A.S. Brun, V. Duez, R.A. García, S. Mathis,

L. Piau, D. Salabert, P.L. Pallé, S.J. Jiménez-Reyes, S. Mathur,

R. Simoniello, and J.M. Robillot

Do Active Regions Modify Oscillation Frequencies?

S.C. Tripathy, K. Jain, and F. Hill

Deep-Focus Diagnostics of Sunspot Structure 378

H. Moradi and S.M. Hanasoge

Are Polar Faculae Generated by a Local Dynamo? 386

K.R. Sivaraman, H.M. Antia, and S.M. Chitre 
The Hanle Effect as Diagnostic Tool for Turbulent Magnetic

Fields

L.S. Anusha, M. Sampoorna, H. Frisch, and K.N. Nagendra

Phase III of the USO Solar Vector Magnetograph

S. Gosain and P. Venkatakrishnan

Revisit of the Classic Wilson Effect 398

S.P. Bagare

The Waldmeier Effect in Sunspot Cycles

B.B. Karak and A.R. Choudhuri

Flare-Driven Acoustic Modes in the Sun

B. Kumar and P. Venkatakrishnan

Dynamics of Active Regions Revealed by Tracking of Doppler

Features

M. Švanda, M. Sobotka, M. Klvaňa, and V. Bumba

The Growth of a Primitive Penumbra

P. Sreejith, A. Tritschler, and K. Sankarasubramanian

Evershed Flow Velocities During 100 Years

K.M. Hiremath

The Thermal Structure of Sunspots

K.M. Hiremath and B.B. Akshatha

Bright Points in G-Band and Ca II H Images from Hinode

C.L. Pradeep and R. Kariyappa

Waves in the Transition Region

E. Scullion, R. Erdélyi, and J.G. Doyle

Viscous Damping of Alfvén Surface Waves with Steady Flows

G.D. Rathinavelu, M. Sivaraman, and A.S. Narayanan

Statistical Detection of Propagating Waves in a Polar Coronal

Hole

G.R. Gupta, E. O'Shea, D. Banerjee, M. Popescu, and J.G. Doyle 
Network Loop Oscillations with EIS/Hinode

A.K. Srivastava, D. Kuridze, T.V. Zaqarashvili, B.N. Dwivedi, and B. Rani

Dynamical Evolution of X-Ray Bright Points with Hinode/XRT 440

R. Kariyappa, B.A. Varghese, E.E. DeLuca, and A.A. van Ballegooijen

Helicity at Photospheric and Chromospheric Heights ...

S.K. Tiwari, P. Venkatakrishnan, and K. Sankarasubramanian

Evolution of Coronal Helicity in a Twisted Emerging Active

Region

B. Ravindra and D.W. Longcope

Power-Law Nanoflare Heating.

L. Prasad and V.K. Joshi

Spectroscopic Diagnostics of Polar Coronal Plumes

K. Wilhelm, B.N. Dwivedi, and W. Curdt

A Flaring Polar Filament

C. Dumitrache

Solar X-Ray Processes

P.C.V. Mallik, J.C. Brown, and A.L. MacKinnon

Time-Varying Thermal Emission in Solar Flares

R. Jain, A.S. Rajpurohit, M. Aggarwal, R. Jamwal, and A. Awasthi

Multi-Wavelength View of Flare Events on 20 November 2003.

P. Kumar, P.K. Manoharan, and W. Uddin

Time-Delay Between Solar Soft X-Ray

and EUV Flare Emissions

M.B. Dhanya and A. Bhardwaj

Major Surge Activity of Super-Active Region NOAA 10484

W. Uddin, P. Kumar, A.K. Srivastava, and R. Chandra

Coronal Magnetic Field Estimation Using Type-II Radio Bursts

K.R. Subramanian, E. Ebenezer, and K.H. Raveesha

Acceleration of CMEs Associated with Eruptive Prominences

A.D. Joshi and N. Srivastava 
Interplanetary Consequences of a Large CME

M. Lahkar, P.K. Manoharan, K. Mahalakshmi, K. Prabhu,

G. Agalya, S. Shaheda Begum, and P. Revathi

Solar System Resonances on Light-Travel Time Scales

Set Up before Proto-Sun's Nuclear Ignition

M.H. Gokhale

Part IV Summaries of Presentations Published Elsewhere

Cycle Prediction from Dynamo Theory

A.R. Choudhuri

Why Does the Torsional Oscillation Precede the Sunspot Cycle?

P. Chatterjee, S. Chakraborty, and A.R. Choudhuri

The Subsurface Magnetic Structure of Solar Active Regions

C.-H. Lin, S. Basu, and L. Li

Sunspot Magnetometry from Kodaikanal

K. Nagaraju, K. Sankarasubramanian, and K.E. Rangarajan

Vector Magnetic Field in Emerging Flux Regions

B. Schmieder and E. Pariat

Evolution of Umbral Dots and Penumbral Grains

M. Sobotka and J. Jurčák

Strong, Localized Downflows in a Sunspot Light Bridge

R.E. Louis, L.R. Bellot Rubio, S.K. Mathew,

and P. Venkatakrishnan

Small-Scale Velocities in Sunspot Penumbrae

M. Franz, R. Schlichenmaier, and W. Schmidt

Photospheric Temperatures from Ca II H

V.M.J. Henriques, D. Kiselman, and M. van Noort

Dual-Line Spectral Imaging of the Chromosphere

G. Cauzzi, K. Reardon, R.J. Rutten, A. Tritschler, and H. Uitenbroek

Inversions of High-Cadence SOLIS-VSM Stokes Observations

C.E. Fischer, C.U. Keller, and F. Snik 
Flows in Flaring and Dormant Active Regions

R.A. Maurya and A. Ambastha

Magnetic and Velocity Field Changes Related to the Solar

Flares of 28 and 29 October 2003

R.A. Maurya and A. Ambastha

A Numerical Investigation of Unsheared Flux Cancelation

J.T. Karpen, S.K. Antiochos, C.R. DeVore, and M.G. Linton

Wave Heating of Coronal Loops with Steady Flows

V.S. Pandey, P. Venkatakrishnan, and A.S. Narayanan

Damping of Prominence Oscillations in Steady Equilibrium

K.A.P. Singh and B.N. Dwivedi

Variation of Network Contrast with Height

K.P. Raju

A Flaring Twisted Emerging Flux Region

R. Chandra, B. Schmieder, G. Aulanier, and J.M. Malherbe

Evidence of Magnetic Reconnection Outflows in a Flare seen

by Hinode/EIS

P. Kumar, A.K. Srivastava, and W. Uddin

Does Coronal Rotation Period Depend on the Sunspot

Number?

H.O. Vats, S. Chandra, and K.N. Iyer

Coronal Magnetic Field from an Extreme Radio Burst

E. Ebenezer, K.H. Raveesha, and K.R. Subramanian

CME Kinematics and Dynamics

C.-H. Lin and P.T. Gallagher

Solar Wind Monitoring with SWIM-SARA Onboard

Chandrayaan-1

A. Bhardwaj, S. Barabash, R. Sridharan, M. Wieser, M.B. Dhanya,

Y. Futaana, K. Asamura, Y. Kazama, D. McCann, S. Varier,

E. Vijayakumar, S.V. Mohankumar, K.V. Raghavendra, T. Kurian,

R.S. Thampi, H. Andersson, J. Svensson, S. Karlsson, J. Fischer,

M. Holmstrom, P. Wurz, and R. Lundin 
Coupling of the Solar Wind and the Magnetosphere 533

Badruddin, V. Gupta, and Y.P. Singh

The FIP Effect in RV Tauri Stars

B.E. Reddy

Author Index.

536 